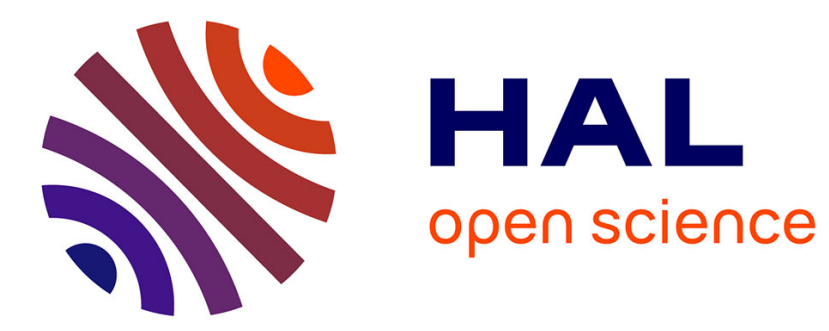

\title{
Alkali clusters: beyond the jellium sphere
}

\author{
Li. Serra, G. Bachelet, N. Giai, E. Lipparini
}

\section{To cite this version:}

Li. Serra, G. Bachelet, N. Giai, E. Lipparini. Alkali clusters: beyond the jellium sphere. Journal de Physique IV Proceedings, 1993, 03 (C7), pp.C7-1965-C7-1969. 10.1051/jp4:19937314 . jpa-00251959

\section{HAL Id: jpa-00251959 https://hal.science/jpa-00251959}

Submitted on 1 Jan 1993

HAL is a multi-disciplinary open access archive for the deposit and dissemination of scientific research documents, whether they are published or not. The documents may come from teaching and research institutions in France or abroad, or from public or private research centers.
L'archive ouverte pluridisciplinaire HAL, est destinée au dépôt et à la diffusion de documents scientifiques de niveau recherche, publiés ou non, émanant des établissements d'enseignement et de recherche français ou étrangers, des laboratoires publics ou privés. 


\title{
Alkali clusters: beyond the jellium sphere
}

\author{
Ll. SERRA, G.B. BACHELET, N.V. GIAI and E. LIPPARINI
}

Dipartimento di Fisica, Università degli Studi di Roma La Sapienza, Piazzale Aldo Moro 2, 00185 Roma, Italy

\begin{abstract}
Ionic pseudohamiltonians, which replace core electrons in atomic calculations, are used to build a new jellium-like model which describes electronic ground state and excitation properties of atomic clusters. As an application, we successfully describe the plasmon resonance in Lithium, Sodium, and Potassium clusters with little additional effort and considerable improvement over the conventional jellium model.
\end{abstract}

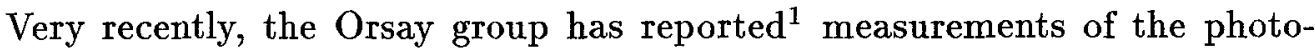
absorption cross section of $\mathrm{Li}$ cluster ions and has pointed out the difficulty to interpret them within the jellium model (JM). Motivated by these results, we present in this work a new model of atomic clusters. We call it the pseudo-jellium model (PJM) because, compared to the conventional JM, ${ }^{2-6}$ it adopts a more accurate description of the electron-ion interactions (the pseudohamiltonian), yet it keeps the conceptual simplicity of the JM and thus allows the self-consistent calculation of ground-state properties and excitation spectra of very large clusters. In what follows we first describe our new model and then show that, unlike the conventional JM, it explains the optical properties of singly ionized alkali clusters.

Calculations of cluster properties are often based on the following electronic energy functional

$$
E=E_{k i n}+E_{x c}+E_{e s}+E_{e i}
$$

where $E_{k i n}, E_{x c}, E_{e s}$, and $E_{e i}$ are the kinetic, exchange-correlation, electrostatic, and electron-ion parts of the total energy of the system of $\mathrm{N}$ valence electrons. In the JM one approximates the real distribution of positive ions by a model ionic density which is constant inside a sphere of radius $R$ and zero outside it

$$
n^{+}(\mathbf{r})=n_{0} \Theta(R-r)
$$

i.e., a spherical step density whose parameters $n_{0}$ and $R$ are related to the WignerSeitz radius $r_{s}$, the number of atoms in the cluster $Z$ and of valence electrons of 
each atom $z_{v}\left(n_{0}=3 / 4 \pi r_{s}^{3} ; R=r_{s}\left(z_{v} Z\right)^{\frac{1}{3}}\right)$, and then assumes that the electronion interaction is simply described by the electrostatic potential due to such a model ionic density

$$
V_{I}(\mathbf{r})=-\int \frac{n^{+}\left(\mathbf{r}^{\prime}\right)}{\left|\mathbf{r}-\mathbf{r}^{\prime}\right|} d \mathbf{r}^{\prime}
$$

The assumption Eq. (3) is very crude and takes no account of the structure of the ions. It evidently amounts to a point-charge approximation of the ionic potential, which cannot resolve any chemical difference between, say, two isovalent ions belonging to the same group of the periodic table. In fact, some of the failures of the $\mathrm{JM}$ in describing correctly the plasmon energies in metal clusters such as $\mathrm{Li}$ clusters ${ }^{1}$ point to this inadequacy of Eq. (3).

It has been shown that the electron-ion interaction can be accurately represented by angular momentum-dependent pseudopotentials ${ }^{7}$ or alternatively by velocity-dependent pseudohamiltonians. ${ }^{8}$ In this work we generalize the JM by substituting the assumption Eq. (3) with the following one

$$
\hat{V}_{I}=\int n^{+}\left(\mathbf{r}^{\prime}\right) h\left(\left|\mathbf{r}-\mathbf{r}^{\prime}\right|\right) d \mathbf{r}^{\prime}
$$

i.e., the ions are still being spherically averaged using the model ionic density $n^{+}$of Eq. (2) (jellium approximation) but, instead of describing the ion-electron interaction by $-1 /\left|\mathbf{r}-\mathbf{r}^{\prime}\right|$, it is now represented by the pseudohamiltonian ${ }^{8}$

$$
h\left(\left|\mathbf{r}-\mathbf{r}^{\prime}\right|\right)=-\frac{1}{2} \nabla_{\mathbf{r}-\mathbf{r}^{\prime}} a\left(\left|\mathbf{r}-\mathbf{r}^{\prime}\right|\right) \cdot \nabla_{\mathbf{r}-\mathbf{r}^{\prime}}+\frac{1}{2} \mathbf{L}_{\mathbf{r}-\mathbf{r}^{\prime}} \frac{b\left(\left|\mathbf{r}-\mathbf{r}^{\prime}\right|\right)}{\left|\mathbf{r}-\mathbf{r}^{\prime}\right|^{2}} \cdot \mathbf{L}_{\mathbf{r}-\mathbf{r}^{\prime}}+v\left(\left|\mathbf{r}-\mathbf{r}^{\prime}\right|\right),
$$

which describes the relative motion of an electron with coordinate $\mathbf{r}$ and an ion at point $\mathbf{r}^{\prime}$. We shall therefore refer to it as the pseudo-jellium model (PJM). In Eq. (5), $a, b, v$ are radial functions and $\mathbf{L}_{\mathbf{r}-\mathbf{r}^{\prime}}$ is the angular momentum operator with respect to the position $\mathbf{r}^{\prime}$. Outside the core of an atom, $a$ and $b$ vanish and $v=-1 /\left|\mathbf{r}-\mathbf{r}^{\prime}\right|$. Inside the core the radial functions $a, b$ and $v$ are determined to optimally replace a full core atom with a pseudo- (valence only) atom. ${ }^{8}$ They are shown in Fig. 1 for the case of the Li atom. From Eqs. $(4,5)$ by integrating over $\mathbf{r}^{\prime}$ one gets

$$
\hat{V}_{I}(r)=-\frac{1}{2} \nabla \alpha(r) \cdot \nabla+\mathbf{L} \beta(r) \cdot \mathbf{L}+u(r),
$$

where $\alpha(r), \beta(r)$, and $u(r)$ are determined from the functions $a(r), b(r)$, and $v(r)$. The modified Kohn-Sham equations for the spherical PJM become

$$
\begin{gathered}
\left\{-\frac{1}{2} \nabla[1+\alpha(r)] \cdot \nabla+\mathbf{L} \beta(r) \cdot \mathbf{L}+u(r)\right. \\
\left.+\int \frac{\rho\left(\mathbf{r}^{\prime}\right)}{\left|\mathbf{r}-\mathbf{r}^{\prime}\right|} d \mathbf{r}^{\prime}+\frac{\delta E_{x c}(\rho)}{\delta \rho}\right\} \varphi_{i}(\mathbf{r})=\epsilon_{\mathbf{i}} \varphi_{\mathbf{i}}(\mathbf{r}) .
\end{gathered}
$$


Note that, since the potential $\hat{V}_{I}$ has the important property of being momentum and angular-momentum dependent, the new Kohn-Sham equations have a modified kinetic energy operator which, just like the case of an isolated ion, can be intepreted in terms of a radial- and direction-dependent effective mass tensor. ${ }^{8}$ The presence of a modified mass has the effect of changing the integrated oscillator strength $m_{1} \equiv \sum_{n} \omega_{n}|<O| D|n>|^{2}$ of the dipole operator $D=\sum_{i=1}^{N} z_{i}$,

$$
m_{1}^{P J M}=\frac{1}{2} \int\left[1+\alpha(r)+\frac{4}{3} r^{2} \beta(r)\right] \rho(r) d \mathbf{r} \equiv \frac{N_{e f f}}{2}
$$

as compared to the JM result

$$
m_{1}^{J M}=\frac{N}{2}
$$

We have evaluated in $\mathrm{Li}_{20}$ the effective number $N_{\text {eff }}$ of valence electrons defined by Eq.(8), finding $N_{\text {eff }}=15$. One can thus interpret the effect of a realistic electron-ion interaction on the dipole excitation as a reduction of the number of valence electrons involved in the collective motion.

As a testing ground of the PJM we choose to apply it to the case of singly ionized alkali clusters. A more detailed discussion of $\mathrm{Li}$ cluster calculations can be found in Ref. 9. Starting from the energy functional we have solved the KohnSham equations Eq. (7) with $r_{s}=3.26,3.93$, and 4.86 a.u. for $\mathrm{Li}, \mathrm{Na}$, and $\mathrm{K}$ clusters, respectively, and calculated the dipole response functions $S(\omega)$ in the time-dependent LDA (TDLDA). ${ }^{10}$ The simple structure of the energy functional makes it possible to reduce the TDLDA problem to a one-dimensional integral equation in coordinate space. In Fig. 2 are shown the calculated photoabsorption cross sections $\sigma(\omega)=\left[4 \pi^{2} \omega / c\right] S(\omega)$ in $\mathrm{JM}$ (dashed) and PJM (solid) for $\mathrm{Li}_{441}^{+}$. The striking feature is the large shift between the two models. Part of this shift can be attributed to the larger single-particle level density of the PJM due to its larger effective mass. The general shape of the distributions is not much changed in the two models. On the same figure are reported the corresponding data of Ref. 1. Although there is some uncertainty in the absolute magnitude of measured cross sections, the peak energies are certainly well determined. Fig. 2 shows a good agreement of the experimental peaks with PJM results whereas the JM predictions are off by about $1 \mathrm{eV}$. In Figs. 3,4 we compare, in atomic units the JM (dashed) and PJM (solid) dipole strength functions for $\mathrm{Na}_{21}^{+}$and $\mathrm{K}_{21}^{+}$. In each case the vertical arrow indicates the measured peak energy. Elementary considerations of pseudopotential theory suggest that if the main idea of this work is correct, then the deviation from conventional jellium spheres must be largest for Li (more nonlocal) and smaller for heavier (less nonlocal) alkali metals. From Figs. 3,4 it clearly appears that such a trend is both experimentally observed and consistently obtained by our calculations: our PJM peak energies are always 
in improved agreement with the experiment when compared to the JM results, whose inadequacy is most evident in the case of Li.

In conclusion, we have shown the importance of the effects of core electrons on valence electrons in alkali metal clusters. These effects can be treated by means of pseudohamiltonians. The resulting pseudo-jellium model keeps the conceptual simplicity of the usual jellium model and leads to a quantitative understanding of optical properties of a wide range of alkali cluster ions.

We are grateful to $\mathrm{C}$. Bréchignac and $\mathrm{Ph}$. Cahuzac for useful discussions. This work was partially supported by the Italian National Research Council (CNR) through Progetto Finalizzato Sistemi Informatici e Calcolo Parallelo under Grants N0. 89.0006.69 and N0. 89.00051.69, and Spanish DGICYT under grant PB89-0332. Division de Physique Théorique of IPN-Orsay is a Unité de Recherche des Universités Paris XI et Paris VI associée au CNRS.

\section{References}

1. C. Bréchignac, Ph. Cahuzac, F. Carlier, J. Leygnier, J. Ph. Roux and A. Sarfati, Contribution to the ISSPIC6, Chicago, Sept. 92, Z. Phys. D to be published; Phys. Rev. Lett. 70, 2036 (1993).

2. W. Ekardt, Phys. Rev. B 31, 6360 (1985).

3. C. Yannouleas, R. A. Broglia, M. Brack and P. F. Bortignon, Phys. Rev. Lett. 63, 255 (1989).

4. G. F. Bertsch, Comput. Phys. Commun. 60, 247 (1990).

5. K. Selby, K. Kresin, V. Masui, J. Vollmer, M. Scheidemann and W. D. Knight, Z. Phys. D 19, 43 (1991), and references therein.

6. C. Bréchignac, Ph. Cahuzac, N. Kebaili, J. Leygnier and A. Sarfati, Phys. Rev. Lett. 68, 3916 (1992) and references therein.

7. D. R. Hamann, M. Schlüter, and C. Chiang, Phys. Rev. Lett. 43, 1494 (1979); G. B. Bachelet, D. R. Hamann, and M. Schlüter, Phys. Rev. B 26, 4199 (1982).

8. G. B. Bachelet, D. M. Ceperley and M.G.B. Chiocchetti, Phys. Rev. Lett. 62,2088 (1989).

9. Ll. Serra, G. B. Bachelet, Nguyen Van Giai, and E. Lipparini, Nota Interna n. 1006, Dipartimento di Fisica, Università di Roma La Sapienza, 19 February 1993.

10. This approximation is sometimes called RPA in other fields, e.g. in nuclear physics, see for example G. F. Bertsch and S. F. Tsai, Phys. Rep. 18 C, 126 (1975).

11. J. Blanc, M. Broyer, J. Chevaleyre, Ph. Dugourd, H. Kuhling, P. Labastie, M. Ulbricht, J.P. Wolf and L. Woste, Z. Phys. D 19, 7 (1991). 


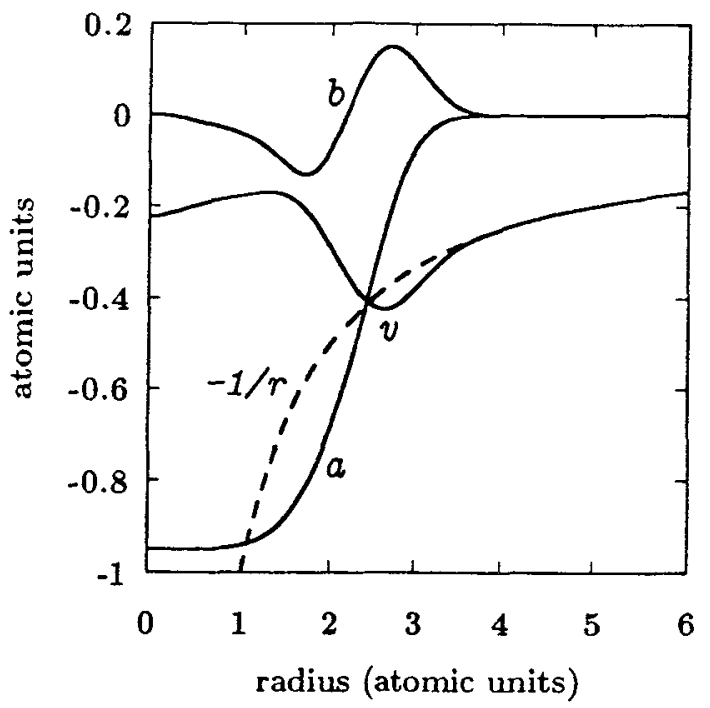

Fig. 1

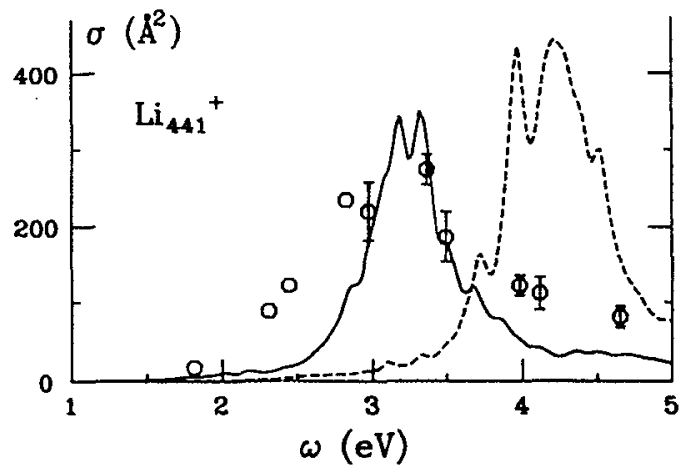

Fig. 2

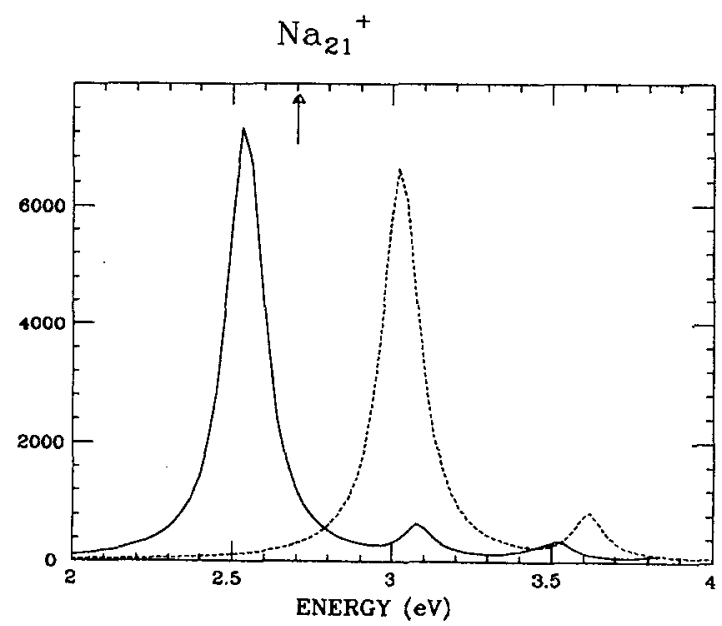

Fig. 3

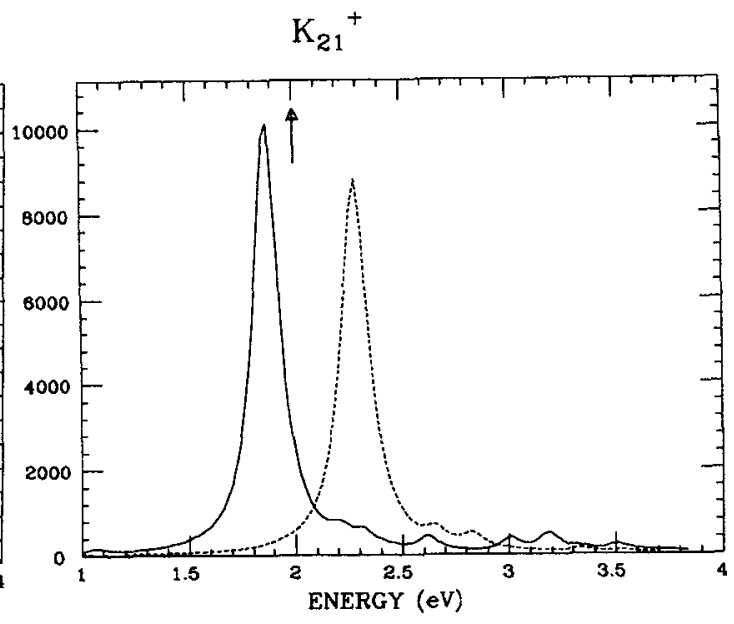

Fig. 4 